\title{
Internal oscillation frequencies and anharmonic effects for the double sine-Gordon
} kink

Salerno, M.; Samuelsen, Mogens Rugholm

Published in:

Physical Review B

Link to article, DOI:

10.1103/PhysRevB.39.4500

Publication date:

1989

Document Version

Publisher's PDF, also known as Version of record

Link back to DTU Orbit

Citation (APA):

Salerno, M., \& Samuelsen, M. R. (1989). Internal oscillation frequencies and anharmonic effects for the double sine-Gordon kink. Physical Review B, 39(7), 4500-4503. https://doi.org/10.1103/PhysRevB.39.4500

\section{General rights}

Copyright and moral rights for the publications made accessible in the public portal are retained by the authors and/or other copyright owners and it is a condition of accessing publications that users recognise and abide by the legal requirements associated with these rights.

- Users may download and print one copy of any publication from the public portal for the purpose of private study or research.

- You may not further distribute the material or use it for any profit-making activity or commercial gain

- You may freely distribute the URL identifying the publication in the public portal 


\title{
Internal oscillation frequencies and anharmonic effects for the double sine-Gordon kink
}

\author{
M. Salerno* and M. R. Samuelsen ${ }^{\dagger}$ \\ Modellering Ikke-Lineaer Dynamik og Irreversibel Termodynamik, The Technical University of Denmark, \\ DK-2800 Lyngby, Denmark \\ (Received 1 July 1988)
}

\begin{abstract}
A simple derivation of the small oscillation frequency around $4 \pi$-kink solutions of the double sine-Gordon equation is presented. Small corrections to these frequencies due to anharmonic effects are also numerically and analytically investigated. The analysis is based on energetic considerations and on the mechanical interpretation of a $4 \pi$ kink as two point particles connected by a spring.
\end{abstract}

\section{INTRODUCTION}

The double sine-Gordon equation (DSG)

$$
\phi_{t t}-\phi_{x x}+\lambda_{1} \sin \phi+\left(\lambda_{2} / 2\right) \sin (\phi / 2)=0,
$$

has received much attention during the past years because of its connection with several physical phenomena such as spin dynamics of superfluid ${ }^{3} \mathrm{He},{ }^{1}$ commensurate-incommensurate phase transitions, ${ }^{2}$ magnetic chains, ${ }^{3}$ domain walls, ${ }^{4}$ etc. In both limits $\lambda_{1}=0$ and $\lambda_{2}=0 \mathrm{Eq}$. (1) reduces to the well-known sine-Gordon equation (SG) with exact soliton solutions. For $\lambda_{1} \neq 0$ and $\lambda_{2} \neq 0$ Eq. (1) has different classes of solitary wave solutions which undergo weakly inelastic scattering. ${ }^{5}$ Among these solutions there is a subclass ( $4 \pi \mathrm{kink}$ ) which can be expressed as linear superposition of two sine-Gordon solitons $^{6}$

$$
\begin{aligned}
\phi\left(x, R_{0}\right)= & 4 \tan ^{-1}\left[\exp \left(-x+R_{0}\right)\right] \\
& +4 \tan ^{-1}\left[\exp \left(x+R_{0}\right)\right]+2 \pi,
\end{aligned}
$$

with $2 R_{0}$ a constant representing the distance between the two sine-Gordon solitons, and $R_{0}$ is related to $\lambda_{1}$ and $\lambda_{2}$ in (1) by

$$
\lambda_{1}=\tanh ^{2} R_{0}, \quad \lambda_{2}=-4 \operatorname{sech}^{2} R_{0} .
$$

From numerical studies on the small oscillation problem around solution (2) it is well known that besides a zero mode frequency (related to translational invariance) there is an additional bound state corresponding to internal oscillations of the two sine-Gordon solitons around the center of mass of solution (2). Slightly different analytical expressions for the frequency of such oscillation have been derived. ${ }^{7,8}$ The aim of the present paper is to present a simple derivation of the small oscillation frequencies around $4 \pi$-kink solutions and to study corrections to these frequencies due to anharmonic effects. The analysis will be based on energetic considerations and on a mechanical analog of solution (2) as two point particles connected by a nonlinear spring. ${ }^{9,10}$ The frequency of the oscillations is then computed in terms of the mass of the particle and of the spring constant of the $4 \pi$-kink oscillator. A numerical investigation of the anharmonic effects of the $4 \pi$-kink oscillations is also performed and com- pared with results obtained by a perturbation analysis. As a result we find the same analytical expression for the small oscillation frequency reported in Ref. 7, while the corrections to these frequencies due to anharmonicity appear to be very small.

The organization of the paper is as follows. In Sec. II we present the derivation of the small oscillation frequency expression while in Sec. III we study both numerically and analytically the corrections to this frequency due to anharmonic effects.

Finally, Sec. IV contains the summary and the conclusions of the paper.

\section{ANALYSIS}

We start by introducing the Hamiltonian for Eq. (1)

$$
\begin{array}{r}
H=\int_{-\infty}^{+\infty}\left\{\frac{1}{2}\left(\phi_{x}^{2}+\phi_{t}^{2}\right)+\lambda_{1}\left(a_{1}-\cos \phi\right)\right. \\
\left.+\lambda_{2}\left[a_{2}-\cos (\phi / 2)\right]\right\} d x,
\end{array}
$$

where $a_{1}$ and $a_{2}$ are suitable normalization constants. Equation (2) is an exact solution of Eq. (1) when $R_{0}$ is a constant and $\lambda_{1}, \lambda_{2}$ are given by Eq. (3). In this case the Hamiltonian (4) acquires the form

$$
\begin{gathered}
H=\int_{-\infty}^{+\infty}\left\{\frac{1}{2}\left(\phi_{x}^{2}+\phi_{t}^{2}\right)+\left(1-\operatorname{sech}^{2} R_{0}\right)(1-\cos \phi)\right. \\
\left.+4 \operatorname{sech}^{2} R_{0}[1+\cos (\phi / 2)]\right\} d x,
\end{gathered}
$$

where the normalization constants $a_{1}, a_{2}$ have been chosen to give zero energy at $\phi= \pm 2 \pi$. In order to characterize small oscillations around solution (2), we observe that for a "wobbler" (i.e., an oscillating $4 \pi$ kink) the distance between the two sine-Gordon solitons oscillates around their static separation $2 R_{0}$. It is therefore natural to assume for such a solution an analytical expression given by Eq. (2) but with $R_{0}$ replaced by a time dependent function $R(t)$ according to

$$
\phi=\phi(x, R(t))=4 \tan ^{-1}[\sinh x \operatorname{sech} R(t)] .
$$

By substituting (6) in (5) and performing the corresponding integral one obtains after some computations

$H\left(R, R_{0}\right)=8[1-(2 R / \sinh 2 R)] \dot{R}^{2}+V\left(R, R_{0}\right)$,

where 


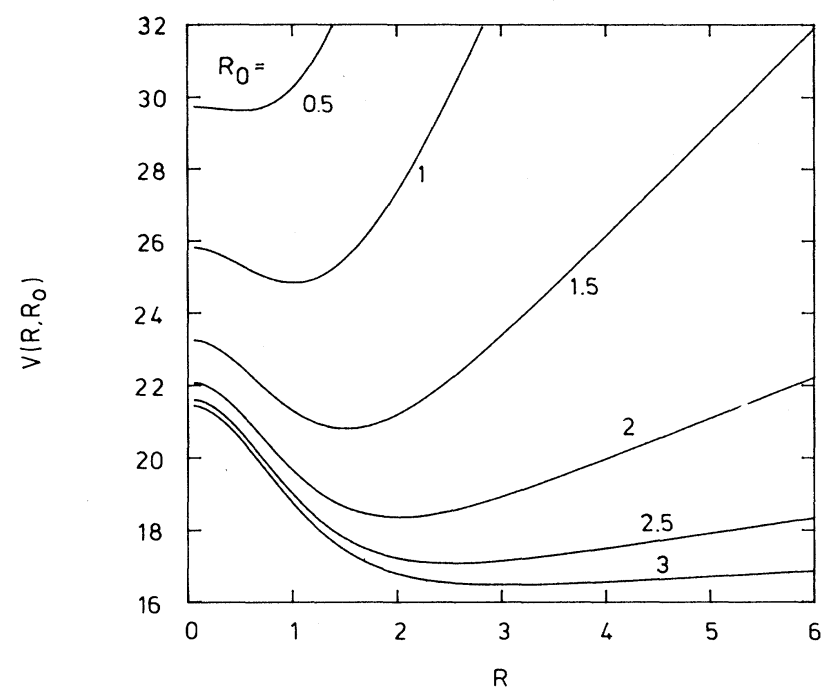

FIG. 1. Different curves of $V\left(R, R_{0}\right)$ vs $R$ for different values of $R_{0}$.

$$
\begin{aligned}
V\left(R, R_{0}\right)= & \left(16 / \tanh ^{2} R\right)[1-(2 R / \sinh 2 R)] \\
+ & \left(8 / \sinh ^{2} R\right)\left[1+\left(\cosh ^{2} R / \cosh ^{2} R_{0}\right)\right] \\
& \times[(2 R / \tanh 2 R)-1] .
\end{aligned}
$$

Equation (7) simply represents the total energy of the wave form (6) expressed as the sum of a kinetic part and a potential one (note the analogy with particles). We also note that in deriving Eq. (7) the only approximation made was the ansatz (6) for the oscillating $4 \pi$ kink. When $2 R$ is equal to the static separation $2 R_{0}$ of the two subsolitons Eq. (7) simply reduces to the rest mass energy of solution (2)

$$
M_{0}=16\left\{1-\left[R_{0} /\left(S_{0} C_{0}\right)\right]\right\},
$$

where $S_{0} \equiv \sinh R_{0}$ and $C_{0} \equiv \cosh R_{0}$.

Introducing the instantaneous mass

$$
M(t)=16\{1-[2 R(t) / \sinh (2 R(t))]\}
$$

Eq. (7) acquires the form

$$
H\left(R, R_{0}\right)=\frac{1}{2} M(t) \dot{R}^{2}+V\left(R, R_{0}\right) .
$$

To study small oscillations around $2 R_{0}$, we let $\left|R-R_{0}\right|<<1$. In this case we can expand the potential energy around $R_{0}$ as

$$
V\left(R, R_{0}\right) \approx V_{0}+V_{0}^{\prime}\left(R-R_{0}\right)+\frac{1}{2} V_{0}^{\prime \prime}\left(R-R_{0}\right)^{2}+\cdots
$$

(here primes denote derivatives with respect to $R$ while the subscript zero means evaluation at $R=R_{0}$ ). From Eq. (8) the first derivative of $V$ is easily calculated as

$$
\begin{aligned}
V^{\prime}\left(R, R_{0}\right)= & 8\left[\left(\cosh ^{2} R / \cosh ^{3} R_{0}\right)-1\right] \\
\times & {\left[2 \cosh ^{3} R \sinh R+\sinh R \cosh R\right.} \\
& \left.\quad-R\left(4 \sinh ^{2} R+3\right)\right] /\left(\sinh ^{4} R \cosh ^{2} R\right),
\end{aligned}
$$

from which one sees that

$$
V_{0}^{\prime}=0 \text {, }
$$

i.e., $R_{0}$ is an extremum for the potential $V\left(R, R_{0}\right)$.

In Fig. $1 V\left(R, R_{0}\right)$ is plotted versus $R$ for different values of $R_{0}$, from which one see that $R_{0}$ is actually a minimum for $V$. (Note also that the potential has a finite value at $R=0$ ).

By inserting Eq. (14) in (12) we get

$$
V\left(R, R_{0}\right) \approx V_{0}+\frac{1}{2} V_{0}^{\prime \prime}\left(R-R_{0}\right)^{2}
$$

and Eq. (11) becomes

$$
H \simeq \frac{1}{2} M_{0} \dot{R}^{2}+\frac{1}{2} V_{0}^{\prime \prime}\left(R-R_{0}\right)^{2}+V_{0}
$$

where we have approximated $M(t)$ with its equilibrium value $M_{0}$ and neglected higher order terms in $R-R_{0}$. Equation (16) is just the energy of a harmonic oscillator in the presence of an external constant potential. The frequency is then evaluated as

$\omega^{2}=3 / S_{0}^{2}-\left\{2\left(S_{0} C_{0}+R_{0}\right) /\left[2\left(C_{0}^{2}\left(S_{0} C_{0}-R_{0}\right)\right]\right\}\right.$,

which is exactly the same expression as is given in Ref. 7.

\section{ANHARMONIC EFFECTS}

In this section we study the corrections to the small oscillation frequency (17) due to anharmonic effects.

To this end we must take into account the fact that the mass of the oscillator is a function of time [see Eq. (10)]. By differentiating Eq. (11) with respect to time and expanding the resulting equation in powers of $\xi=R(t)-R_{0}$ up to third order one gets

$$
\begin{array}{r}
M_{0} \ddot{\xi}+\omega_{0}^{2} \xi+\frac{1}{2} V_{0}^{\prime \prime \prime} \xi^{2}+\frac{1}{2} M_{0}^{\prime}\left(\dot{\xi}^{2}+2 \xi \ddot{\xi}\right)+\frac{1}{2} M_{0}^{\prime \prime}\left(\dot{\xi}^{2}+\xi \ddot{\xi}\right) \xi \\
+\frac{1}{6} V_{0}^{\prime \prime \prime \prime} \xi^{3}=0
\end{array}
$$

Introducing the transformation $\tau=\omega t$ and expanding $\xi$ and $\omega$ in Eq. (18) in powers of $\epsilon$ according to

$$
\begin{aligned}
& \xi=\epsilon \xi_{1}+\epsilon^{2} \xi_{2}+\cdots, \\
& \omega=\omega_{0}+\epsilon \omega_{1}+\epsilon^{2} \omega_{2}+\cdots,
\end{aligned}
$$

one derives from (18) a set of equations (after equating

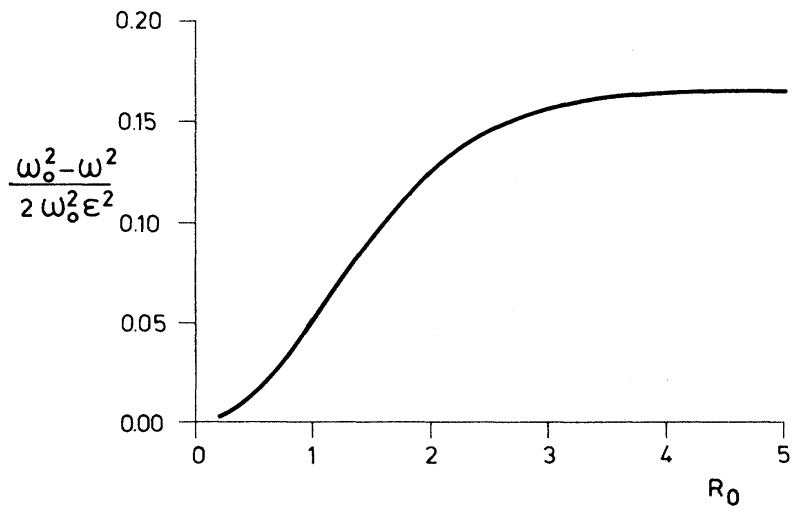

FIG. 2. Oscillatory frequency given by Eq. (20) plotted vs $R_{0}$. 
equal powers of $\epsilon$ ) which can be then solved recursively. After a lengthy but standard computation (see for exam- ple Ref. 11) one finally gets for the frequency the following expression

$$
\left(\omega_{0}^{2}-\omega^{2}\right) / 2 \omega_{0}^{2} \epsilon^{2}=-\frac{1}{16}\left(V_{0}^{\prime \prime \prime \prime} / V_{0}^{\prime \prime}\right)+\frac{1}{8}\left(M_{0}^{\prime \prime} / M_{0}\right)-\frac{1}{16}\left(M_{0}^{\prime} / M_{0}\right)^{2}+\frac{5}{48}\left(V_{0}^{\prime \prime \prime} / V_{0}^{\prime \prime}\right)^{2}-\frac{1}{8}\left(V_{0}^{\prime \prime \prime} / V_{0}^{\prime \prime}\right)\left(M_{0}^{\prime} / M_{0}\right)
$$

where

$$
\begin{gathered}
V_{0}^{\prime \prime \prime}=-16\left[\left(21+28 S_{0}^{2}+4 S_{0}^{4}\right) /\left(C_{0}^{3} S_{0}^{3}\right)\right]+16 R_{0}\left[\left(21+42 S_{0}^{2}+24 S_{0}^{4}\right) /\left(C_{0}^{4} S_{0}^{4}\right)\right], \\
V_{0}^{\prime \prime \prime \prime}=16\left[\left(144+288 S_{0}^{2}+164 S_{0}^{4}+8 S_{0}^{6}\right) /\left(C_{0}^{4} S_{0}^{4}\right)\right]-16 R_{0}\left[\left(144+384 S_{0}^{2}+340 S_{0}^{4}+112 S_{0}^{6}\right) /\left(C_{0}^{5} S_{0}^{5}\right)\right] .
\end{gathered}
$$

In Fig. 2 Eq. (20) is plotted versus $R_{0}$.

To close this section we like to compare this result with a numerical experiment on the anharmonic motion of an oscillating $4 \pi$ kink. To this end we have numerically integrated the DSG equation with $\lambda_{1}, \lambda_{2}$ in (1) given by Eq. (3) for different values of $R_{0}$. The oscillatory motion of a $4 \pi$ kink was started by taking as initial condition expression (6) with $R(t=0)=R_{1}>R_{0}$. The frequency of the resulting oscillation was then computed by following in time the motion of a point on the $4 \pi$-kink profile. (This was checked to be an accurate way to measure the frequency since no radiation (or very little) was generated in the system.

In Figs. 3 and 4 a plot of the resulting frequency versus the amplitude of the oscillation is reported for the values of $R_{0}$, respectively, of 2 and 3. The stars in these figures correspond to numerical results while the solid curves represent the theoretical values predicted by Eq. (20).

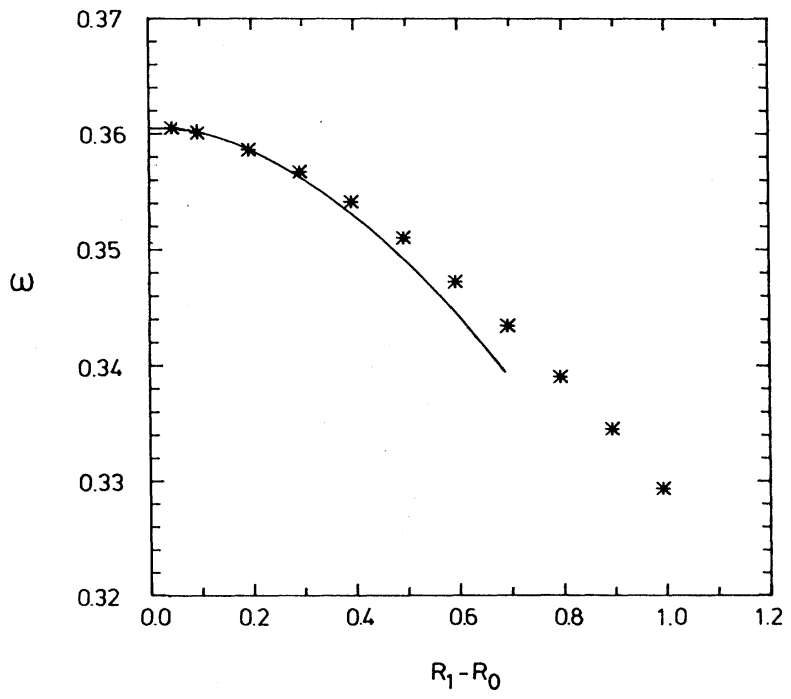

FIG. 3. Oscillatory frequency of a $4 \pi$-"wobbler" vs $\left(R_{1}-R_{0}\right)=\epsilon R_{0}$ for the value $R_{0}=2$. The stars are experimental points while the solid curve corresponds to theoretical values derived from Eq. (20).
We note that the agreement between perturbation theory and experiments is quite good for small values of $R_{1}-R_{0}$. From Figs. 3 and 4 it is also clear that the anharmonicity in this system starts to be relevant only at large oscillation amplitudes.

\section{CONCLUSION}

In the present paper we have given a simple derivation of the small oscillation frequency around $4 \pi$-kink solutions of the double sine-Gordon system. The analysis was based on the assumption (6) for the wave form of suchoscillating solutions.

Finally, a numerical experiment on anharmonic oscillations of $4 \pi$-kink solutions was performed and compared with the predictions of a perturbation treatment. As a result we find a good agreement between the perturbative analysis and the numerical results for small oscillation amplitudes.

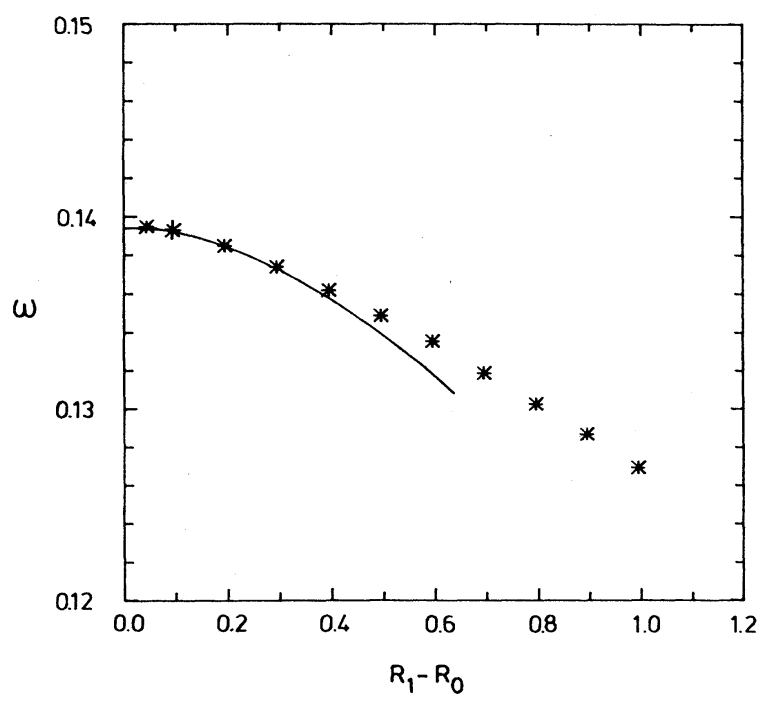

FIG. 4. The same as in Fig. 3 but for $R_{0}=3$. 


\section{ACKNOWLEDGMENTS}

One of us (M.S.) acknowledges financial support from the European Economic Communities through Contract No. St 2-0267-J-C(A), from the European Research Office of the United States Army through Contract No. DAJA-45-85-C-0042, the Thomas B. Thriges Fond (Denmark) and the Gruppo Nazionale di Struttura della Materia, Centro Interuniversitario di Struttura della Materia.
*Also at Laboratory of Applied Mathematical Physics, The Technical University of Denmark, DK-2800 Lyngby. Permanent address: Dipartimento di Fisica Teorica dell'Università, I-84100 Salerno, Italy.

†Also at Physics Laboratory I, The Technical University of Denmark, DK-2800 Lyngby, Denmark.

${ }^{1}$ K. Maki and P. Kumar, Phys. Rev. B 14, 118 (1976); 14, 3290 (1976).

${ }^{2}$ O. Hudak, J. Phys. Chem. 16, 2641 (1983); 16, 2659 (1983).

${ }^{3}$ K. M. Leung, Phys. Rev. B 27, 2877 (1983).

${ }^{4}$ E. Magyari, Phys. Rev. B 29, 7082 (1984).

${ }^{5}$ R. K. Bullough and P. J. Candrey, in Nonlinear Evolution
Equations Solvable by the Spectral Transform, edited by F. Calogero (Pitman, London, 1978), pp. 180-224.

${ }^{6}$ T. Uchiyama, Phys. Rev. D 14, 3520 (1976).

${ }^{7}$ D. K. Campbell, M. Peirard, and P. Sodano, Physica 19D, 165 (1986).

${ }^{8}$ C. R. Willis, M. El-Batanouny, S. Burdick, R. Boesh, and P. Sodano, Phys. Rev. B 35, 3496 (1987).

${ }^{9}$ M. Salerno, Physica 17D, 227 (1985).

${ }^{10}$ M. Salerno and M. R. Samuelsen, Phys. Lett. A 128, 424 (1988).

${ }^{11}$ A. H. Nayfeh, Perturbation Methods (Wiley, New York, 1973). 